Bull. Austral. Math. Soc.

$30 \mathrm{~F} 10,30 \mathrm{C} 20,30 \mathrm{~F} 35,51 \mathrm{M} 10$

VOL. 39 (1989) [369-387]

\title{
UNIFORMISATION OF THE TWICE-PUNCTURED DISC - PROBLEMS OF CONFLUENCE
}

\author{
JoAchim A. Hempel and Simon J. Smith
}

\begin{abstract}
For the twice-punctured unit disc $\Omega_{p}=\{z:|z|<1, z \neq \pm p\}$, where $0<p<1$, we obtain precise descriptions for $p$ near 0 of various parameters associated with the uniformisation of $\Omega_{p}$ by the upper half-plane $U=\{\tau: \operatorname{Im} \tau>0\}$. These parameters include the hyperbolic length of the geodesic surrounding $\pm p$, the so-called "accessory parameters", and the "proximity parameter" which determines the behaviour of the hyperbolic density near the punctures of $\Omega_{p}$.
\end{abstract}

\section{INTRODUCTION}

Let $\left\{p_{1}, \ldots, p_{n}\right\}$ be a set of points of the unit disc $D=\{z:|z|<1\}$, and put $\Omega=D \backslash\left\{p_{1}, \ldots, p_{n}\right\}$. Also, let $U$ denote the upper half-plane $\{\tau: \operatorname{Im} \tau>0\}$, and Möb $U$ the group of all Möbius transformations of $U$ onto itself. By the Uniformisation Theorem there exists a (unique to within an element of Möb $U$ ) conformal universal cover $z=\phi(\tau)$ of $\Omega$ by $U$, and hence a hyperbolic structure on $\Omega$ with metric $\rho(z)|d z|$ defined by

$$
\rho(z)|d z|=(\operatorname{Im} \tau)^{-1}|d \tau|
$$

Further, $\phi$ is automorphic with respect to a Fuchsian group $G$, a subgroup of Möb $U$ without elliptic elements. Now if $n=1$, a universal cover of $\Omega$ by $U$ is

$$
\phi(\tau)=\frac{p_{1}-e^{i r}}{1-\overline{p_{1}} e^{i \tau}}
$$

However, for $n \geqslant 2$ a universal cover cannot be explicitly stated, and, indeed, little quantitative information concerning the various parameters associated with the conformal equivalence of $\Omega$ to $U / G$ appears to be known. In this paper we consider the uniformisation of the twice-punctured disc $\Omega=D \backslash\left\{p_{1}, p_{2}\right\}$, and in particular will be concerned with the situation when $p_{1}$ and $p_{2}$ are close together. Since there exists a number $p, 0<p<1$, and a Möbius transformation $M$ of $D$ onto itself such that $M\left(p_{1}\right)=p, M\left(p_{2}\right)=-p$, we can without loss of generality restrict our study to that of the regions $\Omega_{p}=D \backslash\{ \pm p\}$ where $0<p<1$. We are able to obtain precise descriptions of the behaviour of various parameters associated with the uniformisation of $\Omega_{p}$ by $U$ for $p$ near 0 .

Received 22 July 1988

Copyright Clearance Centre, Inc. Serial-fee code: 0004-9729/89 \$A2.00+0.00. 
There are essentially three parameters whose determination is of particular concern. Firstly, if $\mathcal{F}$ denotes the homotopy class in $\Omega_{p}$ of a circle separating $\{ \pm p\}$ from $\{z$ : $|z|=1\}$, then $\mathcal{F}$ contains a unique hyperbolic geodesic. The length $L$ of this geodesic is the parameter of interest, not only because of its defining geometric interpretation, but also because it is related to trace $T$, the trace of the hyperbolic covering transformation $T$ of $\phi$ determined by $\mathcal{F}$, according to

$$
\mid \text { trace } T \mid=2 \cosh \left(\frac{L}{2}\right)
$$

As a preliminary result, we remark that Theorem 1 of [2] is equivalent to

$$
L=\frac{2 \pi^{2}}{\log \left(8 p^{-1}\right)+o(1)}, \text { as } p \rightarrow 0 .
$$

Although the inverse $\tau=\tau(z)$ of a universal cover of $\Omega_{p}$ by $U$ is multiple-valued, the Schwarzian derivative $\{\tau, z\}$ of $\tau$, defined by

$$
\{\tau, z\}=\left(\frac{\tau^{\prime \prime}}{\tau^{\prime}}\right)^{\prime}-\frac{1}{2}\left(\frac{\tau^{\prime \prime}}{\tau^{\prime}}\right)^{2}
$$

is a single-valued meromorphic function given by

$$
\begin{aligned}
\{\tau, z\}= & \frac{1}{2(z-p)^{2}}+\frac{1}{2(z+p)^{2}}+\frac{1}{2\left(z-p^{-1}\right)^{2}}+\frac{1}{2\left(z+p^{-1}\right)^{2}} \\
& +\frac{m}{z-p}-\frac{m}{z+p}-\frac{p(m p+1)}{z-p^{-1}}+\frac{p(m p+1)}{z+p^{-1}}
\end{aligned}
$$

where $m=m(p)$ is a real-valued constant. $m$ is the so-called accessory parameter at $p$, and is the second of the parameters we will study. Since $z=e^{i \tau}$ is a universal cover of $\{z: 0<|z|<1\}$ by $U$, Hejhal's generalisation of the Carathéodory kernel convergence theorem [1, Theorem 1] shows that $\{\tau, z\} \rightarrow 1 /\left(2 z^{2}\right)$ as $p \rightarrow 0$, uniformly on compact sets in $\{z: 0<|z|<1\}$. Hence a preliminary description of $m$ is given by

$$
m=\frac{-1}{4 p}+o\left(p^{-1}\right), \text { as } p \rightarrow 0 \text {. }
$$

The final parameter we will consider is the quantity $R=R(p)$ determined by the fact that the hyperbolic density $\rho(z)$ on $\Omega_{p}$ satisfies

$$
\rho(z)=\frac{1}{|z-p| \log \frac{1}{R|z-p|}}+o(1), \text { as } z \rightarrow p .
$$

By the symmetry of $\Omega_{p}, \rho(z)=\rho(-z)$, and so

$$
\rho(z)=\frac{1}{|z+p| \log \frac{1}{R|z+p|}}+o(1), \text { as } z \rightarrow-p .
$$

Hence it is sufficient to study $\rho(z)$ near just one of the punctures of $\Omega_{p}$.

Our principal results are stated in the following theorem. 
Theorem 1.1. Suppose $\Lambda=\Lambda(p)$ is defined near 0 by

$$
\Lambda \log \left(\frac{8}{p}\right)+\operatorname{Arg}\left(i \frac{(\Gamma(i \Lambda))^{2}}{\Gamma(2 i \Lambda)}\right)=\frac{\pi}{2}
$$

where the principal value of the argument is taken. Then if

$$
\psi(x)=\frac{\Gamma^{\prime}(x)}{\Gamma(x)}
$$

and

$$
\Delta(x)=\psi(x+1)+\psi(-x)+2 \gamma
$$

where $\gamma$ is Euler's constant, we have as $p \rightarrow 0$

$$
\begin{aligned}
& L=4 \pi \Lambda+O\left(p^{2} \log \frac{1}{p}\right) \\
& m=\frac{-1}{4 p}+\frac{\Lambda^{2}}{p}+O(p)
\end{aligned}
$$

and

$$
\log 2 R=\log \left(\frac{1}{p}\right)+\Delta\left(-\frac{1}{2}+i \Lambda\right)+O\left(p^{2} \log \frac{1}{p}\right)
$$

Remark. Since

$$
\operatorname{Arg}\left(i \frac{(\Gamma(i \Lambda))^{2}}{\Gamma(2 i \Lambda)}\right)=2 \sum_{k=1}^{\infty}(-1)^{k} \frac{\zeta(2 k+1)}{2 k+1}\left(2^{2 k}-1\right) \Lambda^{2 k+1}
$$

where $\zeta(k)$ denotes the Riemann zeta-function $\zeta(k)=\sum_{n=1}^{\infty} n^{-k}$, then (1.5) implicitly defines $\Lambda$ as an infinite power series in $1 / \log \left(8 p^{-1}\right)$. Thus, for example, $m$ is given to within $O(p)$ terms as a series of the form $\sum_{n=0}^{\infty} a_{n} /\left(p\left(\log \left(8 p^{-1}\right)\right)^{n}\right)$, with the first few terms being

$$
m=\frac{-1}{4 p}+\frac{\pi^{2}}{4 p\left(\log \left(8 p^{-1}\right)\right)^{2}}+\frac{\pi^{4} \zeta(3)}{4 p\left(\log \left(8 p^{-1}\right)\right)^{5}}+O\left(\frac{1}{p\left(\log \left(8 p^{-1}\right)\right)^{6}}\right) .
$$

Furthermore, since

$$
\Delta\left(-\frac{1}{2}+i \Lambda\right)=\log \frac{1}{16}+4 \sum_{n=1}^{\infty}(-1)^{n+1} \alpha(2 n+1)(2 \Lambda)^{2 n},
$$


where $\alpha(n)=\sum_{k=1}^{\infty}(2 k-1)^{-n},(1.10)$ defines $R$ to within $O(p \log 1 / p)$ terms as a series of the form $\sum_{n=0}^{\infty}\left(b_{n}\right) /\left(p\left(\log \left(8 p^{-1}\right)\right)^{n}\right)$, with the first couple of terms being

$$
R=\frac{1}{32 p}+\frac{\pi^{2} \alpha(3)}{8 p\left(\log \left(8 p^{-1}\right)\right)^{2}}+O\left(\frac{1}{p\left(\log \left(8 p^{-1}\right)\right)^{4}}\right)
$$

The methods employed to obtain our results are to a large extent techniques from the theory of differential equations. The connection between the uniformisation of $\Omega_{p}$ and differential equations is provided by the observation that if $\tau(z)$ denotes the inverse of a universal cover of $\Omega_{p}$ by $U$, then $1 /\left(\tau^{\prime}(z)\right)^{1 / 2}$ and $(\tau(z)) /\left(\tau^{\prime}(z)\right)^{1 / 2}$ are linearly independent solutions to

$$
\eta^{\prime \prime}+\frac{1}{2}\{\tau, z\} \eta=0
$$

where $\{\tau, z\}$ is given by (1.2).

Conclusions (1.8) and (1.9) of Theorem 1.1 are proved in Section 3; (1.10) is proved in Section 4.

\section{Preliminary Results}

By adapting the method described in Chapter 1 of Nevanlinna [4] for the construction of a fundamental domain for the universal cover of the $n$-fold punctured extended complex plane to the construction of such a domain for the universal cover of $\Omega_{p}$ by $U$, the following lemma is obtained. The proof of the lemma is straightforward once it is noted that if $\phi$ is a universal cover of $\Omega_{p}$ by $U$, and if $\Omega_{p}$ is symmetric with respect to the Euclidean straight line $\Gamma$, then every preimage of $\Omega_{p} \cap \Gamma$ under $\phi$ is a hyperbolic geodesic in $U$, or a finite union of such geodesics.

LEMMA 2.1. There exists a number $\mu>1$ so that the Fuchsian covering group of $\Omega_{p}$ has the fundamental domain $R_{p}$ shown in Figure 1. Apart from the straight line segment $[1, \mu]$, the boundary of $R_{p}$ consists of semi-circles orthogonal to the real axis. The Fuchsian covering group is generated by the transformations $T_{1}$ and $T_{2}$, where

$$
\begin{aligned}
\frac{1}{T_{1}(\tau)+\mu^{1 / 2}} & =\frac{1}{\tau+\mu^{1 / 2}}-\mu^{-(1 / 2)} \frac{\mu^{1 / 2}+1}{\mu^{1 / 2}-1} \\
\frac{1}{T_{2}(\tau)+1} & =\frac{1}{\tau+1}-\frac{\mu^{1 / 2}+1}{\mu^{1 / 2}-1}
\end{aligned}
$$

If $\Omega_{p}^{\prime}=D \backslash(-1, p]$, there exists a univalent function $\tau(z)$ which is a branch of the inverse of a universal cover of $\Omega_{p}$ by $U$, and which maps $\Omega_{p}^{\prime}$ onto the interior of $R_{p}$. As $z$ describes the boundary of $\Omega_{p}^{\prime}$ in the positive direction, passing successively 


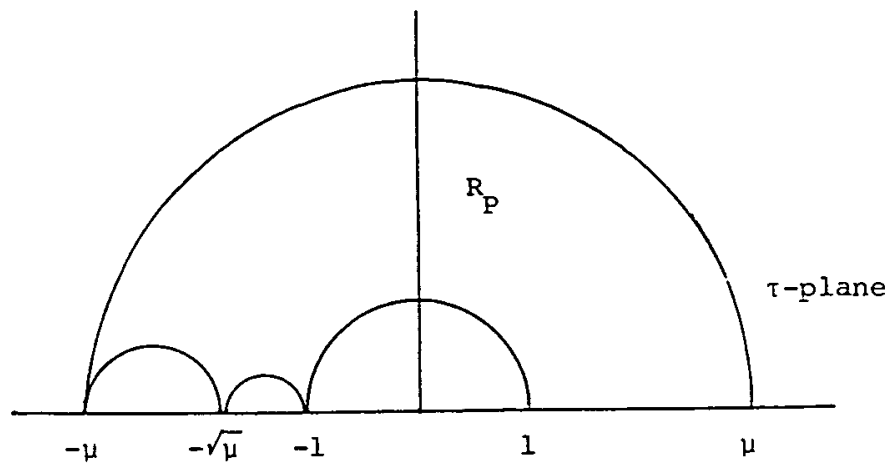

Figure 1. The Fundamental Domain $R_{p}$

through the points $p,-p,-1,-1,-p$ and $p, \tau(z)$ describes the boundary of $R_{p}$, passing successively through the points $-\mu^{1 / 2},-1,1, \mu,-\mu$ and $-\mu^{1 / 2}$, respectively. Further, the straight line segments $(p, 1),(0, i)$ and $(-i, 0)$ in the $z$-plane are mapped by $\tau(z)$ to those segments of $|\tau|=\mu^{1 / 2},|\tau|=\mu^{3 / 4}$ and $|\tau|=\mu^{1 / 4}$, respectively, which lie within $R_{p}$.

The above choice of fundamental domain emphasises the parameter $\mu$, which by a simple geometric argument is shown to be given by $\mu=e^{L}$, where $L$ is the length of the geodesic in the homotopy class $\mathcal{F}$.

The expression (1.2) for $\{\tau, z\}$ is an immediate consequence of the following more general result.

TheOREM 2.2. Let $\left\{p_{1}, \ldots, p_{n}\right\}$ be a finite set of distinct points of $D \backslash\{0\}$, put $\Omega=D \backslash\left\{p_{1}, \ldots, p_{n}\right\}$, and suppose $\tau(z)$ is the (multi-valued) inverse of any universal cover of $\Omega$ by $U$. Then $\{\tau, z\}$ is a single-valued meromorphic function of $z$ given by

$$
\{\tau, z\}=\sum_{k=1}^{n}\left(\frac{1}{2\left(z-p_{k}\right)^{2}}+\frac{1}{2\left(z-1 / \overline{p_{k}}\right)^{2}}+\frac{m_{k}}{z-p_{k}}-\frac{\overline{p_{k}}\left(1+\overline{m_{k} p_{k}}\right)}{z-1 / \overline{p_{k}}}\right)
$$

where the $m_{k}$ are constants, depending only on $\Omega$, which satisfy

$$
\begin{gathered}
\operatorname{Im}\left(\sum_{k=1}^{n} m_{k} p_{k}\right)=0, \\
\sum_{k=1}^{n} p_{k}\left(1+m_{k} p_{k}\right)-\overline{m_{k}}=0 .
\end{gathered}
$$


Proof: It follows from the standard theory of conformal universal coverings that $\{\tau, z\}$ is a holomorphic function on $\Omega \cup\{z:|z|=1\}$, independent of the choice of $\tau(z)$, which near $p_{k}$ can be written as

$$
\{\tau, z\}=\frac{1}{2\left(z-p_{k}\right)^{2}}+\frac{m_{k}}{z-p_{k}}+\psi_{k}(z)
$$

where $\psi_{k}(z)$ is holomorphic at $p_{k}$. Let $\omega=M(z)$ be a Möbius transformation of $\{z:|z|=1\}$ onto $\{\omega: \operatorname{Im} \omega=0\}$, and define $g(\omega)=\tau \circ M^{-1}(\omega)$. From the composition law for the Schwarzian derivative it follows that $\{\tau, z\}=\{g, \omega\}\left(M^{\prime}(z)\right)^{2}$. If $|z|=1$ then $\{g, \omega\}$ is real, and because

$$
\arg \left(M^{\prime}(z)\right)^{2}=-2 \arg (d z)=-2\left(\arg z+\frac{\pi}{2}\right)=-\arg \left(-\frac{1}{z^{2}}\right),
$$

we conclude that $z^{2}\{\tau, z\}$ is real-valued for $|z|=1$. Consequently the function

$$
\psi(z)=z^{2}\{\tau, z\}-\sum_{k=1}^{n}\left(\frac{z^{2}}{2\left(z-p_{k}\right)^{2}}+\frac{z^{2} m_{k}}{z-p_{k}}+\frac{1}{2{\overline{p_{k}}}^{2}\left(z-1 / \overline{p_{k}}\right)^{2}}-\frac{\overline{m_{k}}}{\overline{p_{k}} z\left(z-1 / \overline{p_{k}}\right)}\right)
$$

is holomorphic on the closed unit disc except at 0 , and is real-valued on $\{z:|z|=1\}$. If we define

$$
A=-\sum_{k=1}^{n} \overline{m_{k}}, \quad B=-\sum_{k=1}^{n}\left(\frac{1}{2}+\overline{m_{k} p_{k}}\right),
$$

then near $z=0, \psi(z)$ can be written as

$$
\psi(z)=\frac{A}{z}+B+O(z) .
$$

Also, by the reflection principle, we can write

$$
\psi(z)=\bar{A} z+\bar{B}+O\left(\frac{1}{z}\right)
$$

for $z$ near $\infty$. Thus

$$
K(z)=\psi(z)-\frac{A}{z}-\bar{A} z
$$

is holomorphic on the extended plane, and so is constant. Since $K(0)=B, K(\infty)=\bar{B}$, we obtain (2.2). Further, from $K(z) \equiv B$ we have

$$
\begin{aligned}
\{\tau, z\} & =\frac{\bar{A}}{z}+\frac{B}{z^{2}}+\frac{A}{z^{3}} \\
& +\sum_{k=1}^{n}\left(\frac{1}{2\left(z-p_{k}\right)^{2}}+\frac{m_{k}}{z-p_{k}}+\frac{1}{2 z^{2}{\overline{p_{k}}}^{2}\left(z-1 / \overline{p_{k}}\right)^{2}}-\frac{\overline{m_{k}}}{\overline{p_{k}} z^{3}\left(z-1 / \overline{p_{k}}\right)}\right) .
\end{aligned}
$$

However, $\{\tau, z\}$ is holomorphic at 0 , and so (2.4) can be rearranged to give the identity (2.1), as well as the relation (2.3). 
Remark. If $\Omega=D \backslash\left\{p_{1}=0, p_{2}, \ldots, p_{n}\right\}$, the above proof can be easily modified to show that $\{\tau, z\}$ is of the form

$$
\{\tau, z\}=\frac{1}{2 z^{2}}+\frac{m_{1}}{z}+\sum_{k=2}^{n}\left(\frac{1}{2\left(z-p_{k}\right)^{2}}+\frac{1}{2\left(z-1 / \overline{p_{k}}\right)^{2}}+\frac{m_{k}}{z-p_{k}}-\frac{\overline{p_{k}}\left(1+\overline{m_{k} p_{k}}\right)}{z-1 / \overline{p_{k}}}\right)
$$

where the $m_{k}$ are constants which again satisfy (2.2) and (2.3).

The following lemma will enable us to determine a limiting relation as $p \rightarrow 0$ between the length $L$ of the hyperbolic geodesic in $\mathcal{F}$ and the accessory parameter $m$ as defined by (1.2).

LEMMA 2.3. Let

$$
\text { (i) } \lambda=\frac{L}{4 \pi}, \quad \text { (ii) } c^{2}=\frac{1}{4}(1+2 m p)\left(p^{-2}-p^{2}\right), \quad \text { (iii) } \sigma=\frac{1}{2}\left(p^{-2}+p^{2}\right) \text {. }
$$

Then there exists a non-trivial sequence $\left(a_{n}\right)_{n=-\infty}^{n=\infty}$ of constants which satisfy

$$
\left\{\begin{aligned}
\text { (i) } \limsup _{n \rightarrow \infty}\left|a_{n}\right|^{\frac{1}{n}} \leqslant p^{2} \\
\text { (ii) } a_{n}=\overline{a_{-n}} \text { for all } n \\
\text { (iii) } a_{n-1}\left[(2 n-1+i \lambda)^{2}-\frac{1}{4}\right]-2 a_{n}\left[c^{2}+\sigma\left\{(2 n+i \lambda)^{2}-\frac{1}{4}\right\}\right] \\
\\
\quad+a_{n+1}\left[(2 n+1+i \lambda)^{2}-\frac{1}{4}\right]=0 \text { for all } n
\end{aligned}\right.
$$

Proof: By the reflection principle, the univalent function $\tau(z)$ of Lemma 2.1 can be analytically continued so as to be defined on $V_{p}=C \backslash\left((-\infty, p] \cup\left[p^{-1}, \infty\right)\right)$. Further, reflection across $\left(p, p^{-1}\right)$ gives

$$
\tau(z)=\frac{\mu}{\tau(\bar{z})}, \quad z \in V_{p}
$$

while reflection across the positive imaginary axis gives

$$
\tau(z)=\frac{\mu^{\frac{3}{2}}}{\tau(-\bar{z})}, \quad \operatorname{Im} z>0 .
$$

(2.7) and (2.8) then yield

$$
\tau(z)=\mu^{1 / 2} \tau(-z), \quad \operatorname{Im} z>0
$$

Now, analy tic continuation along a positive circuit about the origin in $p<|z|<p^{-1}$ has the effect of multiplying $\tau(z)$ by $\mu$. Hence, if we consider the principal value of 
$z^{2 i \lambda}$, it follows that $z^{2 i \lambda} \tau(z)$ is single-valued in $p<|z|<p^{-1}$, and so $z^{-2 i \lambda} /\left(\tau^{\prime}(z)\right)$ is holomorphic in this annulus. From (2.9) we deduce that $z^{-2 i \lambda} /\left(\tau^{\prime}(z)\right)$ is odd, and so $z^{-2 i \lambda-1} /\left(\tau^{\prime}(z)\right)$ is a non-zero, holomorphic even function in $p<|z|<p^{-1}$. By requiring that $\left(\tau^{\prime}(-i)\right)^{1 / 2}>0$ and taking the principal value in the numerator, it follows that the square root $h(z)=z^{-i \lambda-1 / 2} /\left(\tau^{\prime}(z)\right)^{1 / 2}$ is holomorphic in $p<|z|<p^{-1}$. Also, $h$ is either odd or even, and since $\arg h(-i)=\arg h(i)=\pi / 4$, we deduce that $h$ is even. If the odd function $Q(z)$ is defined by

$$
Q(z)=\left(z^{2}-p^{2}\right)^{1 / 2}\left(p^{-2}-z^{2}\right)^{1 / 2}, \quad p<|z|<p^{-1}, \quad Q(1)>0
$$

then $(h(z)) /(Q(z))$ is an odd holomorphic function in $p<|z|<p^{-1}$, and so we can write

$$
y_{0}(z)=\frac{1}{Q(z)\left(\tau^{\prime}(z)\right)^{1 / 2}}=z^{i \lambda-\frac{1}{2}} \sum_{n=-\infty}^{\infty} A_{n} z^{2 n}, \quad p<|z|<p^{-1} .
$$

Now $1 /\left(\tau^{\prime}(z)\right)^{1 / 2}$ is a solution to the linear second order differential equation

$$
\eta^{\prime \prime}(z)+\frac{1}{2}\{\tau, z\} \eta(z)=0
$$

If $Q(z)$ is defined by (2.10), the substitution $\eta=Q y$ into (2.11) yields, because of (1.2), the equation

$$
\left[\left(z^{2}-p^{2}\right)\left(z^{2}-p^{-2}\right) y^{\prime}\right]^{\prime}+2\left(z^{2}-c^{2}\right) y=0
$$

which has $1 /\left(Q(z)\left(\tau^{\prime}(z)\right)\right)^{1 / 2}$ as a solution. We deduce that (2.12) has a solution $y_{0}(z)=z^{i \lambda-1 / 2} \sum_{n=-\infty}^{\infty} A_{n} z^{2 n}$ in $p<|z|<p^{-1}$. Substituting the power series for $y_{0}(z)$ into (2.12) gives the recurrence relation

$$
\begin{aligned}
A_{n-1}(2 n+ & i \lambda-3 / 2)\left(2 n+i \lambda-\frac{1}{2}\right) \\
& -2 A_{n}\left[c^{2}+\sigma\left(2 n+i \lambda-\frac{1}{2}\right)\left(2 n+i \lambda+\frac{1}{2}\right)\right] \\
& +A_{n+1}\left(2 n+i \lambda+\frac{1}{2}\right)\left(2 n+i \lambda+\frac{3}{2}\right)=0, \quad \text { for all } n
\end{aligned}
$$

On replacing $n$ by $-n$ in (2.13) we see that $y_{1}(z)=z^{-i \lambda-1 / 2} \sum_{n=-\infty}^{\infty} A_{-n} z^{2 n}$ is a solution to (2.12) in $p<|z|<p^{-1}$, and, on taking conjugates throughout (2.13), we see that 
$y_{2}(z)=z^{-i \lambda-1 / 2} \sum_{n=-\infty}^{\infty} \overline{A_{n}} z^{2 n}$ is a solution to (2.12) in the annulus. Thus $y_{1}(z)$ and $y_{2}(z)$ must be linearly dependent, and so there exists $a \neq 0$ such that $A_{-n}+a \overline{A_{n}}=0$ for all $n$. From this it follows that $|a|=1$, and so the function $z^{i \lambda-1 / 2} \sum_{n=-\infty}^{\infty} a_{n} z^{2 n}$, where $a_{n}=i a^{-1 / 2} A_{n}$ for all $n$, is a solution to (2.12) in $p<|z|<p^{-1}$ with the property that the coefficients $a_{n}$ satisfy (2.6(ii)). The relation (2.6(i)) is then immediate, while (2.6(iii)) follows from (2.13).

By employing results from the elementary theory of infinite continued fractions, we are able to use the sequence $\left(a_{n}\right)$ of Lemma 2.3 to prove the following theorem.

Theorem 2.4. If $m$ and $L$ are defined as in Section 1 , and with $\lambda=L /(4 \pi)$, then

$$
\lim _{p \rightarrow 0} \frac{\left(m p+\frac{1}{4}\right)-\lambda^{2}}{p^{4}}=\frac{25}{32}
$$

Proof: The recurrence relation $(2.6($ iii $))$ can be rewritten as

$$
a_{n}=p_{n} a_{n+1}+q_{n+1} a_{n+2}
$$

where

$$
p_{n}=\frac{2 \sigma\left[(2 n+2+i \lambda)^{2}+\left(\frac{c^{2}}{\sigma}-\frac{1}{4}\right)\right]}{(2 n+1+i \lambda)^{2}-\frac{1}{4}}, q_{n}=\frac{-\left[(2 n+1+i \lambda)^{2}-\frac{1}{4}\right]}{(2 n-1+i \lambda)^{2}-\frac{1}{4}}
$$

Since $p_{n} \rightarrow 2 \sigma$ and $q_{n} \rightarrow-1$ as $n \rightarrow \infty$, we can apply (because of (2.6(i))) Theorem 2.46 of Perron's book [5, p.97] and obtain

$$
\frac{a_{n}}{a_{n+1}}=p_{n}+\frac{q_{n+1}}{p_{n+1}+\frac{q_{n+2}}{p_{n+2}+\frac{q_{n+3}}{p_{n+3}+\frac{q_{n+4}}{\ddots}}}},
$$

valid for $n=0,1,2, \ldots$ Substituting $n=0$ into (2.15), then rearranging, gives

$$
\sigma \frac{a_{1}}{a_{0}}=\frac{1}{2} \frac{(1+i \lambda)^{2}-\frac{1}{4}}{(2+i \lambda)^{2}+\left(\frac{c^{2}}{\sigma}-\frac{1}{4}\right)} \times C(p)
$$


where

$$
C(p)=\frac{1}{1+\frac{q_{1} p_{0}^{-1} p_{1}^{-1}}{1+\frac{q_{2} p_{1}^{-1} p_{2}^{-1}}{1+\frac{q_{3} p_{2}^{-1} p_{3}^{-1}}{1+\frac{q_{4} p_{3}^{-1} p_{4}^{-1}}{\ddots}}}}}
$$

Now, by (1.3) $c^{2} / \sigma \rightarrow 1 / 4$ as $p \rightarrow 0$, and by (1.1) $\lambda \rightarrow 0$ as $p \rightarrow 0$. Hence the quantities $q_{n} p_{n-1}^{-1} p_{n}^{-1}$ approach 0 uniformly in $n$ as $p \rightarrow 0$, and so $C(p) \rightarrow 1$ as $p \rightarrow 0$ (this last statement follows from, for instance, Theorem 38.1 of Wall $[7, p .150]$ ). Hence (2.16) yields

$$
\lim _{p \rightarrow 0} \sigma \frac{a_{1}}{a_{0}}=\frac{3}{32}
$$

However, putting $n=0$ in (2.6(iii)) and $n=0,1$ in (2.6(ii)) leads to the relation

$$
\operatorname{Re}\left[\sigma \frac{a_{1}}{a_{0}}\left\{\frac{1}{\sigma^{2}} \frac{(1+i \lambda)^{2}-\frac{1}{4}}{-\lambda^{2}+\left(\frac{c^{2}}{\sigma}-\frac{1}{4}\right)}\right\}\right]=1 \text {. }
$$

(2.17) and (2.18) then give

$$
\lim _{p \rightarrow 0} \sigma^{2}\left[-\lambda^{2}+\left(\frac{c^{2}}{\sigma}-\frac{1}{4}\right)\right]=\frac{9}{128}
$$

or

$$
\lim _{p \rightarrow 0} \frac{-\lambda^{2}-\frac{1}{4}+\frac{1}{2}(1+2 m p)\left(\frac{1-p^{4}}{1+p^{4}}\right)}{p^{4}}=\frac{9}{32}
$$

(2.14) then follows from (2.19) using (1.3).

We remark that (1.1) and (2.14) can be combined to strengthen (1.3) to

$$
m=\frac{-1}{4 p}+\frac{\pi^{2}}{4 p\left(\log \left(8 p^{-1}\right)\right)^{2}}+o\left(\frac{1}{p\left(\log \left(8 p^{-1}\right)\right)^{3}}\right)
$$

This result is improved considerably in the following section, where the result (2.14) will prove to be of crucial importance. 


\section{A LEGENDRE-TYPE EQUATION}

Instead of working directly with the regions $\Omega_{p}$, in which the punctures depend on $p$, we will find it convenient to work with the regious $B_{p}=\left\{\zeta:|\zeta|<p^{-1}, \zeta \neq \pm 1\right\}$. Then if $S(\zeta)$ denotes the (multi-valued) inverse of any universal cover of $B_{p}$ by $U$, and $z(\zeta)=p \zeta$, we have $\{S, \zeta\}=\{\tau, z\}(d z / d \zeta)^{2}$, where $\{\tau, z\}$ is given by (1.2). Hence

$$
\begin{aligned}
\{S, \zeta\}= & \frac{1}{2(\zeta-1)^{2}}+\frac{1}{2(\zeta+1)^{2}}+\frac{1}{2\left(\zeta-p^{-2}\right)^{2}}+\frac{1}{2\left(\zeta+p^{-2}\right)^{2}} \\
& +\frac{m p}{\zeta-1}-\frac{m p}{\zeta+1}-\frac{p^{2}(m p+1)}{\zeta-p^{-2}}+\frac{p^{2}(m p+1)}{\zeta+p^{-2}}
\end{aligned}
$$

We fix a single-valued branch $S(\zeta)$ of the inverse of a universal cover of $B_{p}$ by $U$ by defining

$$
S(\zeta)=\frac{\mu^{1 / 2}-1}{\mu^{1 / 2}+1} \frac{\tau(p \zeta)-\mu^{1 / 2}}{\tau(p \zeta)+\mu^{1 / 2}}
$$

where $\tau(z)$ is the univalent function of Lemma 2.1. $S(\zeta)$ maps $C \backslash\left((-\infty, 1] \cup\left[p^{-2}, \infty\right)\right)$ univalently onto the infinite strip $-1<\operatorname{Re} S<1$ from which the closed discs with diameters $\left[-1,-\tanh ^{2}(\pi \lambda)\right]$ and $\left[\tanh ^{2}(\pi \lambda), 1\right]$ have been removed. Further, the segments $\left(1, p^{-2}\right)$ and $\left\{p^{-1} e^{i \theta}:-\pi<\theta<\pi\right\}$ in the $\zeta$-plane are mapped by $S(\zeta)$ to the imaginary axis and the line segment $\left(-\tanh ^{2}(\pi \lambda), \tanh ^{2}(\pi \lambda)\right)$, respectively, in the $S$-plane.

With $c^{2}$ defined by $(2.5(i i))$, the differential equation

$$
\left[\left(1-\zeta^{2}\right) Y^{\prime}\right]^{\prime}+\left[\frac{1-2 c^{2} p^{2}}{1-\zeta^{2} p^{4}}-\frac{1-p^{4}}{\left(1-\zeta^{2} p^{4}\right)^{2}}\right] Y=0,
$$

which is obtained by substituting (3.1) into $\eta^{\prime \prime}+1 / 2\{S, \zeta\} \eta=0$, then transforming the equation using $\eta=\left(\zeta^{2}-1\right)^{1 / 2} Y$, has independent solutions

$$
Y_{1}(\zeta)=\frac{1}{\left(\zeta^{2}-1\right)^{1 / 2}\left(S^{\prime}(\zeta)\right)^{1 / 2}}, \quad Y_{2}(\zeta)=\frac{S(\zeta)}{\left(\zeta^{2}-1\right)^{1 / 2}\left(S^{\prime}(\zeta)\right)^{1 / 2}}
$$

in $C \backslash\left((-\infty, 1] \cup\left[p^{-2}, \infty\right)\right)$. In this section we will be concerned only with the solution $Y_{1}(\zeta)$, useful properties of which are summarised in the following lemma.

LEMMA 3.1. The function $Y_{1}(\zeta)$ as defined by (3.4) satisfies

$$
\left\{\begin{array}{l}
\text { (i) } Y_{1}(\zeta) \text { is holomorphic at } \zeta=1 \\
\text { (ii) } Y_{1}(\zeta) \neq 0 \text { for } 1 \leqslant \zeta \leqslant p^{-1} \\
\text { (iii) } \frac{Y_{1}^{\prime}\left(p^{-1}\right)}{Y_{1}\left(p^{-1}\right)}=\frac{-p}{2} \frac{1+p^{2}}{1-p^{2}}
\end{array}\right.
$$


Proof: As $\zeta$ describes a positive loop about $1, S(\zeta)$ is translated by 2 , and so the function $\exp [\pi i S(\zeta)]$ can be written as

$$
\exp [\pi i S(\zeta)]=a_{1}(\zeta-1)+a_{2}(\zeta-1)^{2}+O\left((\zeta-1)^{3}\right), \quad a_{1} \neq 0
$$

Thus

$$
Y_{1}(\zeta)=\left(\frac{\pi i}{2}\right)^{1 / 2}\left[1-1 / 2\left(\frac{a_{2}}{a_{1}}+\frac{1}{2}\right)(\zeta-1)+O\left((\zeta-1)^{2}\right)\right],
$$

and so (i) holds. (ii) is trivial, so it remains to prove (iii). To this end, we note that

$$
\frac{Y_{1}^{\prime}(\zeta)}{Y_{1}(\zeta)}=\frac{-\zeta}{\zeta^{2}-1}-\frac{1}{2} \frac{S^{\prime \prime}(\zeta)}{S^{\prime}(\zeta)}
$$

Now, $S$ satisfies the symmetry conditions

$$
S(\zeta)=-\overline{S(\bar{\zeta})}, S(\zeta)=\overline{S\left(\frac{1}{p^{2} \bar{\zeta}}\right)}
$$

and so

$$
S(\zeta)=-S\left(\frac{1}{p^{2} \zeta}\right)
$$

Differentiating this functional equation twice, then putting $\zeta=p^{-1}$, gives

$$
S^{\prime \prime}\left(p^{-1}\right)=-p S^{\prime}\left(p^{-1}\right)
$$

and then substituting $\zeta=p^{-1}$ in (3.7) yields the required (3.5(iii)).

The function $(2 / \pi i)^{1 / 2} Y_{1}(\zeta)$ is a solution to (3.3) which, in addition to satisfying (3.5), is real-valued on $\left[1, p^{-1}\right]$. We thus can, and will, assume that $Y_{1}(\zeta)$ is real-valued on $\left[1, p^{-1}\right]$. Now, from $(2.14)$ we obtain $2 c^{2} p^{2}=\lambda^{2}+1 / 4+O\left(p^{4}\right)$, and so in $|\zeta| \leqslant p^{-1}$, the equation (3.3) can be written as

$$
\left[\left(1-\zeta^{2}\right) Y^{\prime}\right]^{\prime}+\left[-\lambda^{2}-\frac{1}{4}+O\left(p^{2}\right)\right] Y=0 .
$$

(3.8) is very similar to the Legendre equation

$$
\left[\left(1-\zeta^{2}\right) Y^{\prime}\right]^{\prime}+a(a+1) Y=0
$$

with parameter $a=-1 / 2+i \lambda$. It is the similarity of (3.8) to a Legendre equation which we will exploit. To do this we consider a singular Sturm-Liouville boundary value problem which is suggested by (3.8) and the properties (3.5). The boundary value problem is introduced in the following lemma, where properties of its eigenvalue are also discussed. 
LEMMA 3.2. Consider the Legendre equation

$$
\left[\left(1-\zeta^{2}\right) \phi^{\prime}\right]^{\prime}-\frac{\phi}{4}=\Lambda^{2} \phi
$$

where $\Lambda^{2}$ is determined by the condition that (3.9) has a real-valued solution $\phi_{1}(\zeta)$ which satisfies the conditions

$$
\left\{\begin{array}{l}
\text { (i) } \phi_{1}(\zeta) \text { is holomorphic at } \zeta=1 \\
\text { (ii) } \phi_{1}(\zeta) \neq 0,1 \leqslant \zeta \leqslant p^{-1} \\
\text { (iii) } \frac{\phi_{1}^{\prime}\left(p^{-1}\right)}{\phi_{1}\left(p^{-1}\right)}=\frac{-p}{2} \frac{1+p^{2}}{1-p^{2}}
\end{array}\right.
$$

Then, if $\lambda$ is given by $(2.5(i))$, we can write

$$
\Lambda^{2}=\lambda^{2}+O\left(p^{2}\right)
$$

as $p \rightarrow 0$.

Proof: For convenience, denote the coefficient of $Y$ in $(3.3)$ by $F(\zeta)$. Then from (3.3) and (3.9) we obtain

$$
\left[\left(1-\zeta^{2}\right) Y_{1}^{\prime}\right]^{\prime} \phi_{1}+F(\zeta) Y_{1} \phi_{1}=0
$$

and

$$
\left[\left(1-\zeta^{2}\right) \phi_{1}^{\prime}\right]^{\prime} Y_{1}-\left(\Lambda^{2}+\frac{1}{4}\right) \phi_{1} Y_{1}=0
$$

Subtracting one equation from the other, then integrating from 1 to $p^{-1}$, gives (because of the boundary conditions (3.5) and (3.10))

$$
\int_{1}^{p^{-1}}\left[F(\zeta)+\Lambda^{2}+\frac{1}{4}\right] Y_{1} \phi_{1} d \zeta=0 .
$$

Since $Y_{1} \phi_{1}$ is of constant sign on $\left[1, p^{-1}\right]$, there exists $\zeta_{0} \in\left(1, p^{-1}\right)$ so that $F\left(\zeta_{0}\right)+$ $\Lambda^{2}+1 / 4=0$. As mentioned before, $F\left(\zeta_{0}\right)=-\lambda^{2}-1 / 4+O\left(p^{2}\right)$, and so (3.11) holds.

Note that by Lenma 3.2 we can assume $\Lambda>0$. Furthermore, by (1.1) and (3.11) we have

$$
\Lambda=\frac{\pi}{2 \log \left(8 p^{-1}\right)}+o\left(\frac{1}{\left(\log \left(8 p^{-1}\right)\right)^{2}}\right)
$$

as $p \rightarrow 0$. We are now in a position to prove the key result of this section. 
Theorem 3.3. Supppose $\Lambda>0$ is the parameter defined in the statement of Lemma 3.2. Then as $p \rightarrow 0$ we have

$$
\Lambda \log \left(\frac{8}{p}\right)+\operatorname{Arg}\left(i \frac{(\Gamma(i \Lambda))^{2}}{\Gamma(2 i \Lambda)}\right)=\frac{\pi}{2}+O\left(p^{2} \log \left(\frac{1}{p}\right)\right)
$$

where the principal value of the argument is taken.

PROOF: We begin by noting some properties of solutions to the Legendre equation

$$
\left[\left(1-\zeta^{2}\right) w^{\prime}\right]^{\prime}+a(a+1) w=0
$$

If $F(a, b, c ; \zeta)$ denotes the standard hypergeometric function

$$
F(a, b, c ; \zeta)=1+\sum_{n=1}^{\infty} \frac{(a)_{n}(b)_{n}}{(1)_{n}(c)_{n}} \zeta^{n}
$$

where

$$
(a)_{n}=a(a+1)(a+2) \ldots(a+n-1),
$$

then the solution to (3.14) which is holomorphic at $\zeta=1$ is

$$
P_{a}(\zeta)=F\left(a+1,-a, 1 ; \frac{1}{2}(1-\zeta)\right)
$$

$P_{a}(\zeta)$ can be analytically continued to $C \backslash(-\infty,-1]$. Further, provided that $2 a$ is not an integer, in the part of $C \backslash(-\infty,-1]$ for which $|\zeta|>1, P_{a}(\zeta)$ satisfies

$$
P_{a}(\zeta)=\frac{2^{a}}{\sqrt{\pi}} \frac{\Gamma\left(a+\frac{1}{2}\right)}{\Gamma(a+1)} \zeta^{a} F_{a}(\zeta)+\frac{1}{2^{a+1} \sqrt{\pi}} \frac{\Gamma\left(-a-\frac{1}{2}\right)}{\Gamma(-a)} \frac{1}{\zeta^{a+1}} F_{-(a+1)}(\zeta)
$$

where

$$
F_{a}(\zeta)=1-\frac{a(a-1)}{2(2 a-1)} \zeta^{-2}+\frac{a(a-1)(a-2)(a-3)}{2.4(2 a-1)(2 a-3)} \zeta^{-4}-\ldots
$$

Details of these properties can be found in, for example, Sections 15.4 and 16.1 of Sansone and Gerretsen [6].

It follows from the above comments that we can assume the function $\phi_{1}(\zeta)$ which satisfies (3.9) and (3.10) to be $P_{-1 / 2-i \Lambda}(\zeta)$. The boundary condition $(3.10(\mathrm{iii}))$ and the transition formula (3.16) then yield

$$
\operatorname{Re}\left[\left(\frac{2}{p}\right)^{i \Lambda} \frac{\Gamma(i \Lambda)}{\Gamma\left(\frac{1}{2}+i \Lambda\right)}\left\{p\left(\frac{p^{2}}{1-p^{2}}+i \Lambda\right) F_{-\frac{1}{2}+i \Lambda}\left(p^{-1}\right)+F_{-\frac{1}{2}+i \Lambda}^{\prime}\left(p^{-1}\right)\right\}\right]=0
$$


From the duplication formula for the gamma function we have

$$
\frac{\Gamma(i \Lambda)}{\Gamma\left(\frac{1}{2}+i \Lambda\right)}=\frac{2^{2 i \Lambda-1}}{\sqrt{\pi}} \frac{(\Gamma(i \Lambda))^{2}}{\Gamma(2 i \Lambda)}
$$

and so (3.18) can be written as

$$
\operatorname{Im}\left[e^{i \theta}\left\{p\left(\frac{p^{2}}{1-p^{2}}+i \Lambda\right) F_{-\frac{1}{2}+i \Lambda}\left(p^{-1}\right)+F_{-\frac{1}{2}+i \Lambda}^{\prime}\left(p^{-1}\right)\right\}\right]=0
$$

where

$$
\theta=\Lambda \log \left(\frac{8}{p}\right)+\operatorname{Arg}\left(i \frac{(\Gamma(i \Lambda))^{2}}{\Gamma(2 i \Lambda)}\right)
$$

Now, from (3.12) and (3.17) it follows that $F_{-1 / 2+i \Lambda}\left(p^{-1}\right)=1+O\left(p^{2}\right)$ and $F_{-1 / 2+i \Lambda}^{\prime}\left(p^{-1}\right)=(-3) / 8 p^{3}+O\left(p^{3} / \log (1 / p)\right)$, so (3.19) becomes

$$
\operatorname{Im}\left[e^{i \theta}\left\{\frac{5}{8} p^{3}+i \Lambda p+O\left(\frac{p^{3}}{\log \left(\frac{1}{p}\right)}\right)\right\}\right]=0
$$

or

$$
\cot \theta=\frac{-5 p^{2}}{8 \Lambda}\left(1+O\left(\frac{1}{\log \left(\frac{1}{p}\right)}\right)\right)=O\left(p^{2} \log \left(\frac{1}{p}\right)\right)
$$

Finally, by (3.12) and (3.20) we note that $\theta \rightarrow \pi / 2$ as $p \rightarrow 0$, and upon using the inequality $\left|\cot ^{-1} x-\pi / 2\right| \leqslant|x|$, which is valid for all real $x$, we obtain

$$
\theta=\frac{\pi}{2}+O\left(p^{2} \log \left(\frac{1}{p}\right)\right)
$$

which is $(3.13)$.

From (3.13) we can now establish the results (1.8) and (1.9) of Theorem 1.1. Indeed, from (3.11) we have

$$
\lambda=\Lambda+O\left(p^{2} \log \left(\frac{1}{p}\right)\right)
$$

and since the solutions for $\Lambda$ to (1.5) and (3.13) agree to within $O\left(p^{2}\right)$ terms, (1.8) follows immediately. (1.9) is then a consequence of (2.14). 


\section{THE HYPERBOLIC DENSITY}

Let $S(\zeta)$ denote the single-valued branch of the inverse of a universal cover of $B_{p}=\left\{\zeta:|\zeta|<p^{-1}, \zeta \neq \pm 1\right\}$ by $U$ which was defined by (3.2), and let $\rho^{*}(\zeta)$ be the hyperbolic density on $B_{p}$. In (3.6) we note that $a_{1}>0$, and so near $\zeta=1$ we can write

$$
\rho^{*}(\zeta)=\left[|\zeta-1| \log \frac{1}{a_{1}|\zeta-1|}\right]^{-1}+o(1)
$$

Since $\rho^{*}\left(p^{-1} z\right)=p \rho(z)$, where $\rho(z)$ is the hyperbolic density on $\Omega_{p}$, then

$$
R=p^{-1} a_{1}
$$

where $R$ is the "proximity parameter" defined by (1.4). In this section we use the function $Y_{2}(\zeta)$ of (3.4) to study $a_{1}$, and hence obtain results for $R$.

We begin by noting that $(2 \pi i)^{1 / 2} Y_{2}(\zeta)$, which we will henceforth write as just $Y_{2}(\zeta)$, is a solution to (3.3) which is real-valued on $\left(1, p^{-1}\right]$ and satisfies

$$
\left\{\begin{array}{l}
\text { (i) } Y_{2}(\zeta)-\log (\zeta-1) \rightarrow \log a_{1}, \text { as } \zeta \rightarrow 1 \\
\text { (ii) } Y_{2}(\zeta) \neq 0, \quad 1<\zeta<p^{-1} \\
\text { (iii) } Y_{2}\left(p^{-1}\right)=0
\end{array}\right.
$$

Guided by this, we consider the Legendre equation

$$
\left[\left(1-\zeta^{2}\right) \phi^{\prime}\right]^{\prime}-\frac{\phi}{4}=\Lambda^{2} \phi
$$

where $\Lambda^{2}$ is determined by the condition that (4.3) has a solution $\phi_{2}(\zeta)$, real-valued on $\left(1, p^{-1}\right]$, which satisfies the same three properties $(4.2)$ as does $Y_{2}(\zeta)$. As in Lemma 3.2 , we obtain

$$
\Lambda=\lambda+O\left(p^{2} \log \left(\frac{1}{p}\right)\right)
$$

and so $\Lambda$ is given to within $O\left(p^{2} \log (1 / p)\right)$ terms as the solution to (1.5).

We need to further discuss properties of solutions to the Legendre equation (3.14). If we use the notation of Hille [3,pp.307-311], then a solution at $\zeta=1$ to (3.14), independent of the function $P_{a}(\zeta)$ given by $(3.15)$, is the function $P_{a, 1}(\zeta)$ defined by

$$
\begin{aligned}
P_{a, 1}(\zeta) & =P_{a}(\zeta) \log \frac{1}{2}(1-\zeta)+\sum_{n=1}^{\infty} \frac{(a+1)_{n}(-a)_{n}}{(1)_{n}(1)_{n}} \\
& \times \sum_{j=0}^{n-1}\left[\frac{1}{a+1+j}+\frac{1}{-a+j}-\frac{2}{1+j}\right]\left[\frac{1}{2}(1-\zeta)\right]^{n}
\end{aligned}
$$


where the principal branch of the logarithm is used. $P_{a, 1}(\zeta)$ is initially defined in $\{|\zeta-1|<2\} \backslash(1,3)$, but can be analytically continued to $C \backslash((-\infty,-1] \cup[1, \infty))$. Our purposes require a continuation of $P_{a, 1}(\zeta)$ which is defined on $(1, \infty)$, and for this reason we define $P_{a, 1}^{*}(\zeta)$ to be the right-hand side of (4.4), where the logarithm is given by $0<\arg 1 / 2(1-\zeta)<2 \pi$. Then $P_{a, 1}^{*}(\zeta)$ is initially defined on $\{|\zeta-1|<2\} \backslash(-1,1)$, but can be continued to $C \backslash(-\infty, 1]$. At $\zeta=\infty,(3.14)$ has linearly independent solutions $Q_{a}(\zeta)$ and $Q_{-1-a}(\zeta)$, where

$$
Q_{a}(\zeta)=\frac{\Gamma\left(\frac{1}{2}\right) \Gamma(a+1)}{\Gamma\left(a+\frac{3}{2}\right)}(2 \zeta)^{-1-a} F\left(\frac{a}{2}+\frac{1}{2}, \frac{a}{2}+1, a+\frac{3}{2} ; \zeta^{-2}\right)
$$

$Q_{a}(\zeta)$ and $Q_{-1-a}(\zeta)$ can be continued so as to be single-valued on $C \backslash(-\infty, 1]$. We will need a formula for $P_{a, 1}^{*}(\zeta)$ in terms of $Q_{a}(\zeta)$ and $Q_{-1-a}(\zeta)$. Such a formula can be gleaned from Hille - the details are presented in the following lemma.

Lemma 4.1. With $P_{a, 1}^{*}(\zeta), Q_{a}(\zeta)$ and $Q_{-1-a}(\zeta)$ defined in $C \backslash(-\infty, 1]$ according to the above discussion, then

$$
\begin{aligned}
P_{a, 1}^{*}(\zeta)=[ & \left.-1-\Delta(a) \frac{\tan \pi a}{\pi}+i \tan \pi a\right] Q_{a}(\zeta) \\
& +\left[-1+\Delta(a) \frac{\tan \pi a}{\pi}-i \tan \pi a\right] Q_{-1-a}(\zeta),
\end{aligned}
$$

provided that $2 a$ is not an integer. Here $\Delta(a)$ is defined by (1.6) and (1.7).

Proof: From (4.5) we have

$$
Q_{a}(-\zeta)=-e^{i a \pi} Q_{a}(\zeta), \quad \operatorname{Im} \zeta>0,
$$

and from the definitions of $P_{a, 1}(\zeta)$ and $P_{a, 1}^{*}(\zeta)$ it follows that

$$
P_{a, 1}^{*}(\zeta)=P_{a, 1}(\zeta)+2 \pi i P_{a}(\zeta), \quad \operatorname{Im} \zeta>0 .
$$

Now by Hille $[3$, p.309] we have

$$
P_{a, 1}(\zeta)=\frac{\pi}{\sin \pi a} P_{a}(-\zeta)-\Delta(a) P_{a}(\zeta)
$$

and so by (4.8) we deduce

$$
P_{a, 1}^{*}(\zeta)=\frac{\pi}{\sin \pi a} P_{a}(-\zeta)+(2 \pi i-\Delta(a)) P_{a}(\zeta), \quad \operatorname{Im} \zeta>0 .
$$

However, by Hille $[3, p .310]$ we have

$$
P_{a}(\zeta)=\frac{\tan \pi a}{\pi}\left[Q_{a}(\zeta)-Q_{-1-a}(\zeta)\right]
$$


and then (4.6) follows for $\operatorname{Im} \zeta>0$ (and hence for $\zeta \in C \backslash(-\infty, 1]$ ) by substituting for $P_{a}(-\zeta)$ and $P_{a}(\zeta)$ in (4.9) using (4.10), and then applying (4.7).

We can now describe the behaviour of $a_{1}$ as $p \rightarrow 0$.

Proof of (1.10): The function $\phi_{2}(\zeta)$, which is a solution to (3.14) with $a=$ $-1 / 2+i \Lambda$, satisfies $\phi_{2}(\zeta)-\log (\zeta-1) \rightarrow \log a_{1}$ as $\zeta \rightarrow 1$, and so

$$
\phi_{2}(\zeta)=P_{-\frac{1}{2}+i \Lambda, 1}^{*}(\zeta)+\left(\log 2 a_{1}-i \pi\right) P_{-(1 / 2)+i \Lambda}(\zeta)
$$

Because $\phi_{2}\left(p^{-1}\right)=0$, we can use the transition formulae (4.6) and (4.10) to obtain

(4.11) $\log 2 a_{1}=\Delta\left(-\frac{1}{2}+i \Lambda\right)-i \pi \tanh \pi \Lambda\left(\frac{Q_{-(1 / 2)+i \Lambda}\left(p^{-1}\right)+Q_{-(1 / 2)-i \Lambda}\left(p^{-1}\right)}{Q_{-(1 / 2)+i \Lambda}\left(p^{-1}\right)-Q_{-(1 / 2)-i \Lambda}\left(p^{-1}\right)}\right)$

Now, $Q_{a}(\zeta)$ can be written as

$$
Q_{a}(\zeta)=\frac{\sqrt{\pi}}{2^{a+1}} \frac{\Gamma(a+1)}{\Gamma\left(a+\frac{3}{2}\right)} \frac{1}{\zeta^{a+1}} F_{-(a+1)}(\zeta)
$$

where $F_{a}(\zeta)$ is defined by (3.17) (see Sansone and Gerretsen [6, pp.555-6]). Thus, by using the duplication formula for the gamma function, (4.11) becomes

$$
\log 2 a_{1}=\Delta\left(-\frac{1}{2}+i \Lambda\right)-\pi \tanh \pi \Lambda \frac{\operatorname{Re}\left[e^{-i \theta} F_{-(1 / 2)-i \Lambda}\left(p^{-1}\right)\right]}{\operatorname{Im}\left[e^{-i \theta} F_{-(1 / 2)-i \Lambda}\left(p^{-1}\right)\right]}
$$

where

$$
\theta=\Lambda \log \left(\frac{8}{p}\right)+\operatorname{Arg}\left(i \frac{(\Gamma(i \Lambda))^{2}}{\Gamma(2 i \Lambda)}\right)
$$

Since $\Lambda=\lambda+O\left(p^{2} \log (1 / p)\right)$, we conclude from (1.8) that

$$
\theta=\frac{\pi}{2}+O\left(\left(p \log \left(\frac{1}{p}\right)\right)^{2}\right)
$$

and so

$$
\log 2 a_{1}=\Delta\left(-\frac{1}{2}+i \Lambda\right)+O\left(p^{2} \log \left(\frac{1}{p}\right)\right)
$$

(1.10) then follows from (4.1). 


\section{REFERENCES}

[1] D.A. Hejhal, 'Universal covering maps for variable regions', Math. $Z$. 137 (1974), 7-20.

[2] J.A. Hempel and S.J. Smith, 'Hyperbolic lengths of geodesics surrounding two punctures', Proc. Amer. Math. Soc. 103 (1988), 513-516.

[3] E. Hille, Lectures on Ordinary Differential Equations (Addison-Wesley, London, 1969).

[4] R. Nevanlinna, Analytic Functions (Springer-Verlag, Berlin, 1970).

[5] O. Perron, Die Lehre von den Kettenbrüchen, Band II (B.G. Teubner Verlagsgesellschaft, Stuttgart, 1957).

[6] G. Sansone and J. Gerretsen, Lectures on the Theory of Functions of a Complex Variable, Vol. II (Wolters-Noordhoff, Groningen, 1969).

[7] H.S. Wall, Analytic Theory of Continued Fractions (Chelsea, Bronx N.Y., 1967).

Assoc. Professor J.A. Hempel

Department of Mathematics Statistics and Computing Science

University of New England

Armidale, N.S.W., 2351

Australia
Dr S.J. Smith

Department of Mathematics

Bendigo College of Advanced Education

P.O. Box 199

Bendigo, Victoria, 3550

Australia 